\title{
652 ANTI-PD-L1/IL-15 KD033 ACTIVATED MACROPHAGES AND INDUCED ANTI-TUMOR IMMUNITY IN THE TUMOR- MICROENVIRONMENT
}

Stella Martomo*, George Wang, Zhanna Polonskaya, Xenia Luna, Dan Lu, Jeegar Patel. Kadmon Corporation, New York, NY, United States

Background KD033 is a clinical-stage bispecific fusion molecule consisting of a high-affinity anti-human-PD-L1 antibody and human IL-15. Previous studies with KD033 in mice expressing functional human PD-1 and PD-L1 showed that KD033 was efficacious in reducing the growth of both PD-L1 positive and negative tumors. ${ }^{1}$ In the microenvironment of PD-L1 negative tumors, PD-L1 would still be expressed by some immune cells such as macrophages. The goal of the current study is to evaluate direct effect of KD033 on macrophages through in vitro studies and assess its contribution to anti-tumor immunity.

Methods Monocyte-derived human macrophages were treated with either KD033, the non-targeting IL15 fusion counterpart (ntKD033) or anti-PD-L1 antibody in vitro, and the supernatants were analyzed for cytokine/chemokine secretion. HumanPD-1/PD-L1 transgenic C57BL/6 mice subcutaneously transplanted with the human-PD-L1 positive or negative MC38 colon carcinoma were treated with KD033 intravenously when tumors reached $100 \mathrm{~mm}^{3}$. Tumor infiltrating cell populations were evaluated with Immunohistochemistry (IHC).

Results In vitro cultures with KD033 induced macrophages to secrete inflammatory cytokines such as IFN $\gamma$ to a much higher level compared to ntKD033 cultures at the same concentrations. The anti-PD-L1 antibody alone did not induce macrophages to secrete IFN $\gamma$. IHC on KD033-treated human PD-1/ PD-L1 transgenic mice showed that PD-L1 negative and positive MC38 tumors have similar levels of CD8 T cell tumor infiltrations. IHC with the macrophage marker CD68 showed higher level of CD68/IFN $\gamma$ double positive cell infiltrations on PD-L1 negative tumors that was correlated with increased tumor growth inhibitions.

Conclusions Increased in vitro IFN $\gamma$ secretion from KD033treated macrophages correlated with increased CD68/IFN $\gamma$ double positive cell infiltrations in PD-L1 negative MC38 tumors from KD033-treated human PD-1/PD-L1 transgenic mice as evaluated by IHC. We hypothesized that our anti-PDL1/IL15 KD033 induces anti-tumor immunity in PD-L1 negative tumors by activating PD-L1-expressing immune cells such as macrophages in the tumor microenvironment.

\section{REFERENCE}

1. Martomo S, et al. Single-Dose anti-PD-L1/IL-15 fusion protein KD033 generates synergistic antitumor immunity with robust tumor-immune gene signatures and memory responses. Mol Cancer Ther 2021;20(2):347-356.

Ethics Approval Mouse studies were conducted for Kadmon by Wuxi AppTec Inc. All the procedures related to animal handling, care and the treatment in the study were performed according to the guidelines approved by the Institutional Animal Care and Use Committee (IACUC) of WuXi AppTec following the guidance of the Association for Assessment and Accreditation of Laboratory Animal Care (AAALAC).

http://dx.doi.org/10.1136/jitc-2021-SITC2021.652 